\title{
Integration of IN and Internet: The Balance between Idealism and Current Products Realism
}

\author{
Geir Gylterud ${ }^{1}$, Michael Barry ${ }^{2}$, Valerie Blavette ${ }^{3}$, Uwe Herzog $^{4}$, Telma Mota ${ }^{5}$ \\ ${ }^{1}$ Telenor R\&D, Otto Nielsens v.12, \\ 7004 Trondheim, Norway \\ ${ }^{2}$ Broadcom Eireann Research Ltd., Kestrel House, Clanwilliam Place, \\ Dublin 2, Ireland \\ ${ }^{3}$ CNET-DAC/ARP, Technopole Anticipa, 2 avenue Pierre Marzin, \\ 22307 Lannion Cedex, France \\ ${ }^{4}$ T-Nova, Technologiezentrum, \\ D-64307 Darmstadt, Germany \\ ${ }^{5}$ Portugal Telecom Inovação, Rua Eng. José F. Pinto Basto, \\ 3810 AVEIRO, Portugal \\ geir.gylterud@telenor.com \\ mab@broadcom.ie \\ valerie.blavette@cnet.francetelecom.fr \\ uwe.herzog@telekom.de \\ telma@ptinovacao.pt
}

\begin{abstract}
Since the beginning of the 90's Intelligent Networks (IN) have been deployed in telecommunication networks worldwide for the realisation of value-added services in circuit switched networks. In today's marketplace, evolutionary trends in IN and the growth of the Internet, have the potential to make a wide range of advanced services available to consumers. To facilitate this, a new service architecture providing an environment for the development of integrated IN-Internet services is proposed here. This new service architecture combines the best parts of IN and the Internet. It will evolve from current industry initiatives and developments and from existing products focussed on the integration of IN and Internet such, that today's gateways and switches will provide access to tomorrows services and platforms.
\end{abstract}

\section{Introduction}

The business environment for classical telecom / IN services is undergoing rapid change. New trends of both technical nature and social character have emerged in the area of telecommunications. Firstly, there is a clear trend for distribution of telecom hardware and software over the network. Secondly, deregulation in the telecom market is driving the opening network interfaces to other service providers and competitors. Lastly, the success of the Internet means that it is becoming a big competitor for the classical telephone network and IN.

More and more customers have access to the Internet and are being provided with new Internet based services. Some of these services are developed by Internet service 
providers and are multimedia and interactive in nature, others are typical telephony services for which the Internet is used as transport media, e.g. IP telephony. Huge growth in the IP telephony market is forecast in the near future. According to a Frost \& Sullivan report the IP telephony revenues are predicted to rise from 260 million US\$ in 1998 to 13,8 billion US\$ in 2005 [1]. This and similar examples [2] underline the shift from circuit switched networks towards packet switched networks. In some countries two years ago the amount of packet traffic already exceeded the circuit switched traffic.

Despite the growth of IP based telephony services, IN services will continue to exist for several years. IN has already solved problems that Internet is still fighting with, including quality, availability, reliability and the creation of revenue. A practical solution is to converge IN and the Internet in order to exploit the benefits they offer and compensate their weaknesses. Such an integrated IN-Internet architecture enables new classes of service that are characterized by a mix of Internet and Telecom functionality, using the synergy of both. These integrated services will make use of the ubiquitous telephony / IN service and of the user friendliness and openness of the Internet, providing wide ranges of choice and value to the consumer. The introduction and provision of these new services over heterogeneous networks (PSTN and Internet) requires generic but practical solutions for the evolution of Network Intelligence. In particular, solutions must be provided for the following questions:

1. How should the PSTN/IN and Internet be connected?

2. How can a joint service platform spanning IN and Internet services be realised?

3 . How can $3^{\text {rd }}$ party service providers be given access to the network preserving network integrity?

EURESCOM project P909, running from January 1999, is investigating answers and solutions for the above issues of IN-Internet convergence. These investigations focus on a common Reference Architecture, defined by the project, and implemented on middleware-based on off-the-shelf products and/or prototypes. In particular the project addresses:

- new IN services and integrated IN-Internet services

- evolution of IN architecture (Business Model and architecture elements)

- use of existing concepts to evolve IN towards an open architecture (e.g. TINA) and to define open APIs (e.g. á la Parlay)

- control of special resources (e.g. VoIP gateways, PABXs, e-mail servers, etc.)

- investigation and integration of existing products/prototypes from the telecom and IT industry

This paper presents solutions to the questions posed above. It starts by introducing a Reference Architecture for IN-Internet integration. Following this, some of the main features and components to be developed for the architecture are described. An overview of existing technologies and products in the area of IN-Internet integration that are being integrated into the reference architecture is presented. Finally some conclusions on state-of-the art for IN/Internet integration are drawn. 


\section{Putting IN and Internet together}

In general interworking between the PSTN and Internet can be broken into two main areas:

1. Interworking at bearer control level,

2. Interworking at service control level.

In the case of bearer control there has been a lot of work in developing protocols and standards within the ITU and the IETF. Voice over the Internet (VoIP) is now widely commercially available using TCP/IP and new protocols such as H.323 [3]. Such internetworking is facilitated by a gateway function which translates between the internet and PSTN bearer protocols. Another protocol which is currently being standardised by IETF, for the control of media gateways, is the Media Gateway Control protocol (MeGaCo)[4].

With respect to Service and service control there are quite a number of reasons for communicating at this level:

- Providing end-to-end service over a heterogeneous PSTN/VoIP call

- Providing IN services to internet users,

- Facilitating an internet entity (e.g. web server) to access data in the PSTN,

- Facilitating an internet entity to start a call in the PSTN.

- To allow web-based customer-control access to an IN service.

- Facilitating the IN to access internet data (e.g. directory information)

The interface to the existing IN systems may be at the SMF level for non-callrelated purposes such as user service profile modifications, at the SCF level for setting up PSTN calls or at the SSF level by using basic IN switching capabilities and triggering mechanisms.

This paper proposes the integration at the service control level through the definition and implementation of a unique service platform, able to support joint services spanning both IN and the Internet. A Reference Architecture has been developed, utilising features of existing initiatives and products in its specification and implementation. The following sections describe in more detail the realisation of the platform.

\section{The Reference Architecture}

The architectural framework is presented in Fig.1. It identifies three distinct layers:

- the service control layer (Service Platform) providing telecommunication-related services and service features, e.g., IN-like services; such services can be either offered/sold to end users or third party service providers that belong to a different domain;

- the resource layer, which contains all the network (public and private) and special resources controlled by the control and service components;

- the adaptation layer contains adapters that interface the service control platform functionality with the underlying resource layer. 
The different layers inter-operate through well defined and public API's and/or protocols) and a Distributed Processing Environment (DPE), which provides the communication capabilities among the functionality that are supported within the service platform.

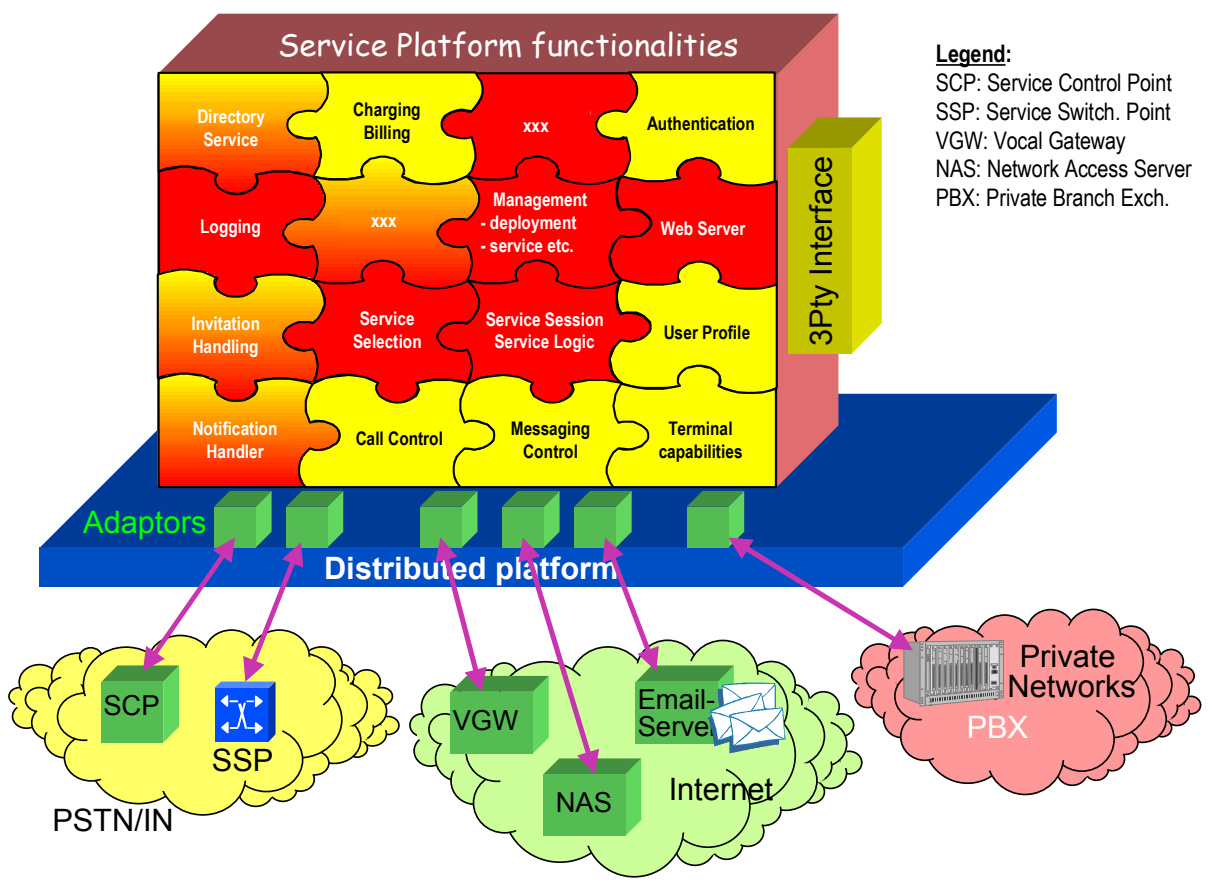

Fig. 1. Architectural framework

Fig. 1 depicts also a 3Pty interface offered by the platform to $3^{\text {rd }}$ parties in an external domain; e.g. consumers or third party providers. In some cases, the external domain will support a subset of the functionality supported by the service platform, such as subscription or accounting functionality. In other cases, it will support only service specific functionality and rely on the service platform for all the service generic functionality. The platform is flexible enough to offer to third parties different kinds of services and APIs at different levels. The functionality of platform should be used "on-demand".

The service control layer is a collection of functionality that interacts over the DPE. Composition and interaction of functionality allows service designers to build added value telecommunication services, while being abstracted away from the underlying network and special resources. In addition, this provides "service features" to services and applications in the external domain. These service features are offered by means of secured/controlled interfaces in order to ensure secure, robust and accountable interactions. 
Particular interest is given to the Call control component in the service platform. This component offers a common call control interface to all services and provides the mechanisms to control calls across heterogeneous networks. Similarly, the Message Control component provides an abstract interface to services in the Service Control Layer. It maps towards messaging equipment and special peripherals providing support for e.g. email, fax, text-to-speech, speech recognition and other features.

The network and special resources can activate services implemented in the services layer by means of events that are sent to the service platform. Resource adapters, or wrappers, are used to abstract the upper layer from the details of specific protocols (e.g. INAP, Megaco/H.248).

To ensure that the service and network architecture is versatile enough to fulfil the needs and requirements identified for integrated IN-Internet services; it has been validated through the specification of use cases and services scenarios comprising of Click-to-Dial, Internet Call Waiting, Distributed \& Enhanced Call-Centre, Virtual Presence, Universal Messaging and Meeting Scheduler services.

\section{Major Components of the Reference Architecture}

To build the proposed architecture and APIs existing concepts, specifications and products from existing standards bodies and initiatives are reused, particularly Parlay[5] and TINA[6].

The Parlay Industry Working Group has specified a new, open, network Application Programming Interface that allows new applications and services to be developed and deployed on today's and tomorrows networks[7]. The API is technology and network independent. It helps the network operator to maximise the value of their network technology by directly passing on that functionality to third parties in a safe and controlled manner, whilst hiding much of the complexity of network signalling from the developer.

The work of the TINA Consortium has also been reused in the platform TINA has defined and validated a consistent and open architecture for distributed telecommunications software applications [8]. Useful TINA concepts include the independence of service and transport networks, integration of control and management, adoption of object orientation and distributed processing techniques and reuse of existing standards wherever possible. Using TINA principles services can be developed and deployed in a network independent manner, making it a desirable candidate architecture for the integration of PSTN and Internet networks.

The service platform is composed of typical TINA components and other components above a Parlay-like interface. These present a common view of the communication capabilities typically offered by IN and other networks, allowing the service platform to be deployed over heterogeneous network environments. The main components that abstract the service platform from the underlying networks are the "Call Control", "User Interaction Control" and "Message Control" components. These components, depicted in Fig.2, offer a set of abstract operations to be used by 
advanced telecommunications services. They can be used to access all networks and special resources in the underlying network.

Authentication, Service Selection, Invitation Handling and the Service Session/Logic are also interesting functionality from the architecture point of view. These "service features" may be offered to third parties as part of the service platform.

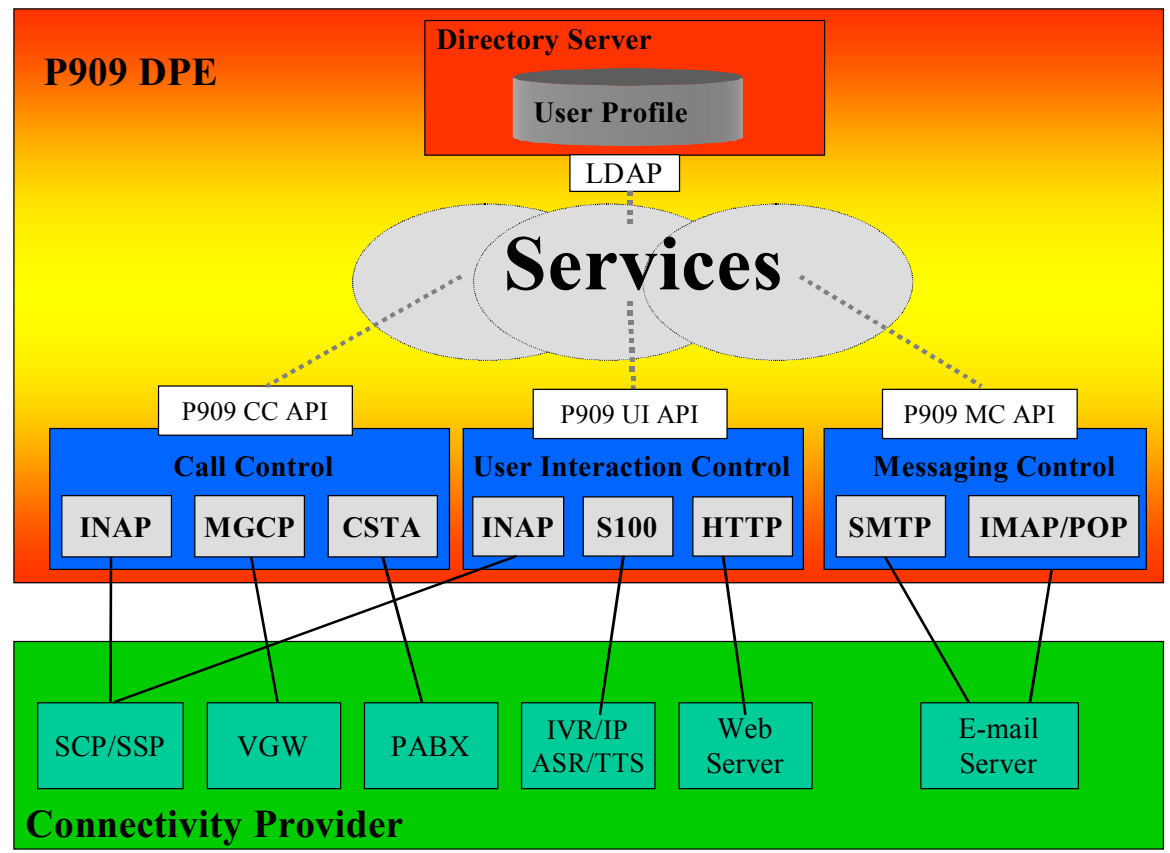

Fig. 2. Major components of the service platform

\subsection{Call Control, Messaging Control and User Interaction}

The Call Control component offers a common call control interface to all service applications. The definition of this Call Control interface is strongly based on Parlay. The Call Control component is responsible for the mapping between the set of operations offered to the services and the protocol specific operations supported by the underlying networks. It forwards notifications of new incoming calls to the service if a request has been made for such Notification. Call Control acts as a mediator for hybrid call legs connected to actors from different networks domains, such as the PSTN and Internet. This applies to all calls that require some intelligent routing to be executed and where call legs of different types are involved.

The Call Control functionality has two parts. A generic Call Control functionality providing services and a generic call control API with adapters towards different networks/protocols. The generic part includes a call and session model, simplified so 
that it can cover the features of the call/session model common to all underlying networks. This call/session model is, if needed, refined in the adapter for each underlying network. These adapters also translate internal operations to the protocol dependent operations needed to control network resources. The resources covered include: SCP, SSP, VGW and PBX. The supported protocols consist of, amongst others, INAP, CSTA, MeGaCo, SIP and proprietary SCP interfaces.

The Message Control component provides an abstract interface to services in the Service Control Layer. It maps towards messaging equipment and special peripherals providing support for e.g. voicemail, email, fax, SMS and other features. It forwards notifications of new incoming messages to the service if a request has been made for such notification. The Messaging Control like the Call Control is divided into two parts: generic and adaptation. The generic part is responsible of providing services with a generic messaging API and adapters are also needed here towards different messaging systems involved comprising of servers for e-mail, voice-mail, fax and SMS.

The User Interaction Control component provides an abstract User Interaction interface to the service components. It is responsible for mapping between the set of operations offered to the services and the protocol specific operations supported by the underlying network resources. It supports functionality for playing messages, collecting information, speech recognition and text-to-speech translation. As the other components this component is divided in two parts: A generic User Interaction functionality and adapters towards different resources. The resources may be IVR, ASR and TTS equipment and Web-servers supporting protocols like INAP, S100 and HTTP.

\subsection{User Profile}

The User Profile component stores an individual user profile for each user of a service. It contains service independent information like name and address, service specific information in the form of a user service profile for each subscribed service and registration information e.g. for UPT like services. Each user has his individual service profile for each service they are a user of. Service profiles contain information about constraints imposed by the provider or retailer such as restrictions according to service contract and the subscriber e.g. credit limits, restrictions in service options as well as user preferences and other selectable options e.g. language, user level.

The User profile is stored in a Directory Server and the services use LDAP to access the information stored in a users profile.

\section{Supporting IN through Interworking}

Interworking the service platform with IN poses unique problems. In particular, IN does not support separation between access, usage and communication or the separation between transport and the service architectures. To help solve this problem, the TINA-IN WG of the TINA-C have specified a TINA-IN interworking unit between the SSP and TINA[9]. This IWU, called the ITAU (IN-TINA adaptation 
UNIT) acts both as a consumer, to represent an IN user for access and services sessions, and as a connectivity provider, to enable the setup of usage sessions over the IN. The principles of the ITAU have been adopted, with modifications in the platform. While the TINA-IN WG provides a mechanism to allow the setup of a TINA access session on behalf of an IN subscriber, it introduces some problems with service selection for IN services. Normally service selection in IN is done at the beginning of a call when the service code or destination address is first dialled. Later, if needed a PIN is introduced e.g. for Credit Card Calling. The PIN may vary from service to service. Additionally the calling Id (i.e. CLI) may or may not be sent. In TINA the procedure is different; first, a User name and a Password are requested and only then the access to the provider services is offered. In summary, the idea of having an access session was to allow the user to access to the Retailer domain and not to a specific service Furthermore, not all IN services require this type of Access, for many anonymous access is sufficient.

The TINA-IN WG solution proposes that the ITAU decides if a named or anonymous access session is required. This implies that, the Interworking function knows which service is to be invoked and decides what type of access is required. A better solution is to introduce additional TINA Access functionality in the consumer domain to decide whether or not to instantiate an access session depending on some criteria (e.g. type of service, terminal or network). This functionality can be kept

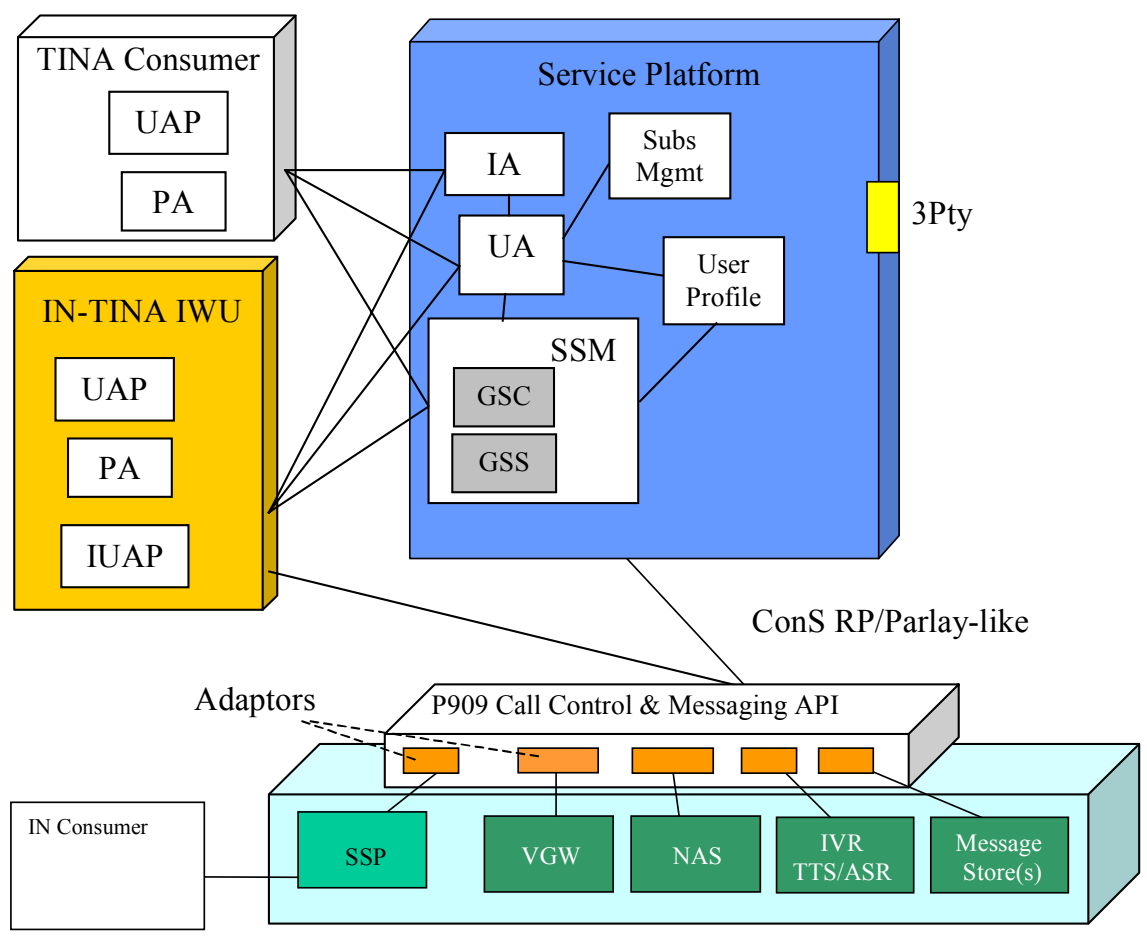

Fig. 3. TINA and the reference Architecture for IN Interworking 
separate from the existing, specified TINA functionality in the consumer domain in order to maintain the service independence of the existing consumer access functions.

As with the architecture described in this paper, the TINA-IN WG have adopted Parlay for the control and usage of calls in the IN network. While the Parlay Call Control Services are used in similar ways in both TINA-IN and here, there are some differences in how they are viewed. Rather than viewing the entire IN as a single terminal and using the Parlay interlace towards that terminal, this reference architecture places Parlay at the interface between the retailer and the connectivity provider, at the ConS reference point. This greatly simplifies the setup of calls and other services across multiple network types.

Fig.3 presents a TINA view of the reference architecture. It includes TINA components to implement the functionality of the reference architecture and the interworking towards IN.

\section{Products and Prototypes}

In order to complete the needed architecture functionality existing products and prototypes from the telecommunications and information technology have been used, together with the TINA and Parlay based components. A survey of existing products and technologies with capabilities to enable IN-Internet integration has been performed and the results has been used in defining the prototyping and experimental phases of the P909 project. The survey also provides a snapshot of the state-of-the-art in the area of IN-Internet integration as realised using available products and technologies.

Examining the high-level architecture to be used in the project identified technology classifications. Products related to telephony networks; internet based networks; services network; service management and the DPE. Products and technologies in each of these areas have been evaluated for openness, flexibility and usefulness in the provision of advanced services.

In telephony networks, access to network functions and services through the adoption of open interfaces allows greater flexibility in the provision of advanced services, especially for third party service providers. Industry Initiatives such as JAIN $^{\mathrm{tm}}[10]$ and Parlay are driving the opening up of the PSTN network by providing open environments and interfaces for the development of advanced services. Private network interfaces, such as TAPI and JTAPI can be used for advanced service provision in PBX based networks.

Within the PSTN, SS7 and INAP remain core technologies that must be interfaced towards to insure the smooth integration of the PSTN with other network types, particularly the Internet. Companies such as Ericsson, Lucent, Radvision and Dialogic are building operational PSTN-Internet gateways to provide interworking to the PSTN at a switching infrastructure level.

Technologies for the provision of telephony and conferencing services over the Internet continue to be studied by both the ITU and the IETF. These studies have resulted in, respectively, the H.323 and SIP protocols that have been widely adopted. Currently many telecoms and IT vendors, including Dynamicsoft, Microsoft, Cisco and Radvision have products that support these protocols. H.323 Gateways are the most common means of interworking the PSTN and Internet for voice and multimedia 
communications. A single H.323 gateway is used for both the communications protocol (H.323) and to control the interworking between the PSTN and the Internet The IETF and ITU are jointly studying a protocol, Megacop/H.248, for the control of media gateways from the Internet, independently of any higher-level communications protocol.

Before a new service can be offered it must be designed, implemented and deployed. Object oriented technologies and methodologies, including UML and OMT, have been used in the development of sample services in the project by the use of different tools that utilise these methodologies.

The DPE is a central part of the project infrastructure. The DPE environment is seen not only as one option for a runtime environment but also as the basis for the integration of IN/PSTN and Internet within the project. DPEs based on CORBA, DCOM and Java have been utilised in the experiments.

The following table identifies the key technologies used by the partners in the project for building experimental environments and services.

Table 1. Key technologies for the provision of integrated IN-Internet services

\begin{tabular}{|l|l|}
\hline Area & Technology \\
\hline Analysis \& Design & UML, OMT, SDL \\
\hline $\begin{array}{l}\text { Service Creation \& } \\
\text { Execution }\end{array}$ & IN platforms, DCOM Active X, JavaBeans \\
\hline Public Network Interfaces & SS7/ INAP, Parlay, JAIN \\
\hline Private Network Interfaces & JTAPI, TAPI, CSTA, \\
\hline DPE Environments & CORBA, DCOM, RMI \\
\hline Network Access & ISUP/Radius/TACACS+ \\
\hline $\begin{array}{l}\text { Internetworking \& } \\
\text { Media Gateways }\end{array}$ & $\begin{array}{l}\text { H.323, ISUP/H.323, SIP, PINT, SGCP, IPDG, MGCP, } \\
\text { MeGaCo }\end{array}$ \\
\hline Terminals & $\begin{array}{l}\text { Personal JAVA Platform, Windows CE, PDAs, WAP } \\
\text { terminals }\end{array}$ \\
\hline Messaging services & X.400, X.500, SMTP, IMAP, POP, LDAP, TUP \\
\hline WWW services & HTML, XML, ASP, Javascript, Java Applets \\
\hline
\end{tabular}

It should be noted that not all of the technologies listed in the above table have been used in operational environments. Some of them are for experimental use only; others would need some additional development and customisation to make them full products. A more detailed description of these technologies is available in [11]. 


\section{Conclusions}

Hybrid services which exploit the synergy of IN and the Internet will soon be a reality. However, as yet, there are no formal mechanisms for the development and deployment of such services. A practical approach for the specification and implementation of an integrated IN-Internet service platform will utilise existing functionality and equipment, as well as introduce advanced service concepts from the IT and Telecoms industries. In particular, products, solutions and platforms that are distributed, object oriented and support separation of service access and usage and separation of transport and service networks are desirable features in any future service platform.

This paper has described such a service platform and identified major components, technologies and initiatives needed for the development of the platform. In particular it has described call control, messaging and user interface components that are necessary to abstract services and applications away from the operation of the underlying networks. As well as these, components for user profile, service access and interworking with IN has been identified.

Assembling the service platform also requires that existing state-of-the-art products and technologies are integrated. Existing gateway solutions from IT and Telecom vendors have been used to provide access to services from PSTN and Internet terminals. Furthermore, it has been possible to reuse a wide range of, primarily Internet based products, for security, directory and user services.

The design of the service platform, including the internal and external interfaces has been validated using a set of service scenarios representing advanced services, including Click-to-Dial, Internet Call Waiting, and Unified Messaging. These services are currently being implemented for the service platform within the Eurescom P909 project.

\section{References}

1. Frost \& Sullivan Report 3650 "The European Market for Internet Protocol"

2. Forrester Research; "Internet Telephony Grows Up"; March 1997

3. ITU-T Rec. H.323, "Packet based multimedia communication systems", 1998

4. Fernando Cuervo et al; "MeGaCo Protocol"; draft-ietf-megaco-protocol-03.txt; ftp://standards.nortelnetworks.com/megaco/docs/Oslo99/megacoHGCP12.pdf

5. Parlay Consortium; http://www.parlay.org

6. TINA Consortium; http://www.tinac.com

7. Parlay Consortium, Parlay API specification, version 1.2, 10 September 1999

8. TINA Consortium; TINA service architecture", Version 5.0, L. Kristiansen et al, June 1997.

9. TINA Consortium; TINA-IN Work Group RfP "IN access to TINA services \& Connection management"; 14 October 1999

10. http://java.sun.com/products/jain

11. "What an IN system should be", M Barry et al., EURESCOM P909 Deliverable 1, http://www.eurescom.de 


\section{Acknowledgements}

The information presented in this paper is based on the work done in the EURESCOM project P909-GI "Enabling Technologies for IN Evolution and INInternet Integration". This however does not imply that the paper necessarily reflects a common position of all involved EURESCOM Shareholders/Parties. The authors would like to thank their colleagues working on EURESCOM P909 for their insightful comments. 